\title{
QUALITY OF LIFE IN CONSTRUCTION SITE WORKERS
}

\author{
SUKHADA PRABHU ${ }^{\mathrm{a} 1}$, DARSHANA JAIN ${ }^{\mathrm{b}}$, MANASI DESAI ${ }^{\mathrm{c}}$ \\ a Department of Kinesio therapeutics and Physical Diagnosis, D.Y. Patil University, School of Physiotherapy, Nerul, Navi Mumbai, \\ Maharashtra, India

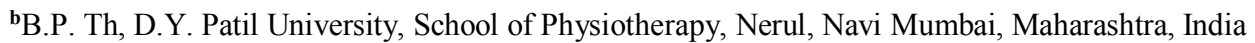 \\ ${ }^{\mathbf{c}}$ Department of Neuro Physiotherapy, D.Y. Patil University, School of Physiotherapy, Nerul, Navi Mumbai, Maharashtra, India
}

\begin{abstract}
In India, the Construction Industry is the second largest and a fast growing sector. Occupational hazards are one of the major problems faced by construction workers.Construction workers suffer from musculoskeletal pain, respiratory conditions, skin diseases due to longer working hours and exposure to various chemicals. They not only suffer from physical health problems but also from emotional distress and psychological ill health as they work in poor conditions and are often vulnerable to exploitation. This study mainly aims to understand the quality of life of construction workers in different domains using SF 36questionnaire.
\end{abstract}

KEYWORDS: Occupational hazards, Quality of life, Construction site workers

Occupational health plays an important role because one third of the time is spent in working place. Heal their ergonomic advice plays a very important role in promoting good occupational health. Construction site workers job requires standing for long hours, their job involves carrying weight like concrete, bricks etc. on head and shoulder, walking with the weight for a particular distance as well as climbing stairs. In construction site, main activities involves excavation, masonry works, vehicle movement or driving, plastering, working at height, painting, spraying, cutting, grinding bricks and removing of debris. They also load and unload the weight in an inappropriate way or in an abnormal posture which leads to increase stress on the body due to faulty biomechanics. They work for 8-10hours a day for the whole week, so considering it to be physically very demanding job.

Construction workers are continuously exposed for many types of work related hazards at the worksite. These dangers or hazards can be physical (noise, vibration and machine grinding); chemical (gases, dust and fumes); biological (bacteria, viruses and fungi) and ergonomic hazards (awkward postures, bending and insufficient rest breaks). Workers work in poor lightning, poor ventilation, Conjunction of work place. They also work in extreme weather conditions and lack personal protective equipment's (PPE) or have insufficient PPE or substandard PPE. Exposure to physical agents like asbestos, silica, organic solvents, sewer gases, welding fumes, radiation noise \& vibration result in acute injury, chronic illness, permanent disability and various health problems and risks like lung diseases such as common cold, asthma, cough, chronic bronchitis, emphysema and occupational lung diseases. Construction workers do not have health care benefits and often these factors lead to poor quality of life. Due to stressful hours at work, maladaptive behaviour like drinking, smoking, overeating are very common in them. Unstable jobs, low income also leads to psychological distress in workers which results in poor quality of life.

Quality of life is a multidirectional construct incorporating an individual's subjective perception of physical, emotional and social wellbeing including both a cognitive component and emotional component. There are various scales to measure quality of life like generic or disease specific quality of life scales out of which SF 36 is most reliable $\&$ valid tool. Reliability and validity testing of SF 36 questionnaire for evaluation of quality of life of Chinese urban construction workers was studied in 2009 which concluded that SF 36 has high reliability in determination of quality of life among urban construction workers. SF 36 questionnaire is a multipurpose, short form health survey with only 36 questions, it yields as an 8 scale profile of functional health and wellbeing scores as well as psychometrically based physical and mental health summary measures and a preference based health utility index. SF 36 helps in evaluating individual patient's health status, researching the cost effectiveness of a treatment and monitoring and comparing disease burden. 


\section{SCOPE OF THE STUDY}

This study mainly aims to understand the quality of life of construction workers in different domains and main objective is to determine the level of work stress affecting workers general health, their physical health, emotional health and social activities and to put forward measures to improve quality of life in construction workers. The paucity of literature measuring quality of life for labourers is the need of the study.

\section{MATERIALS AND METHODS}

A cross sectional survey was carried out among 60 construction site workers in age group of 18-60 years. Prior Informed consent was taken from the construction site workers. Demographic details was administered with a proforma and SF 36 questionnaire was administered to workers in an interview format.

\section{RESULTS AND DISCUSSION}

A survey was conducted and total 60 construction workers were taken as subjects. Construction workers had not only limitations in physical functioning but complains of various emotional and psychological problems and loss of energy which could be attributed to less social functioning and functional capacity.

\begin{tabular}{|c|c|}
\hline Demographic Details & Mean \pm SD \\
\hline Age & $20 \pm 5$ \\
\hline Years Worked & $15 \pm 4$ \\
\hline Working Hours & $15 \pm 4$ \\
\hline
\end{tabular}

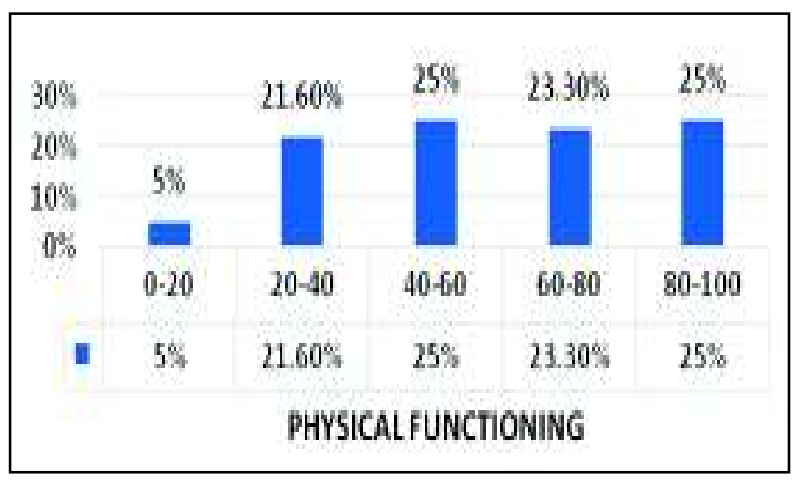

Graph 1: Physical Functioning

Inference

In the above graph it shows that $25 \%$ workers $(80-$ 100) had no limitations in physical functioning, $23.8 \%$ workers (60-80) had mild to no affection of physical functioning, 25\% workers (40-60) had mild limitations, $21.6 \%$ workers $(20-40)$ had moderate limitations while $5 \%$ percent (0-20) workers showed severe affection of limitations of physical functioning.

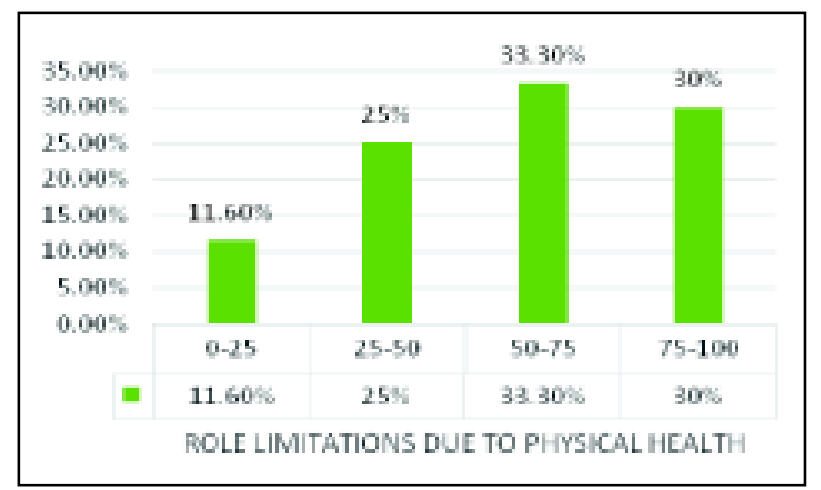

Graph 2: Role Limitations Due to Physical Health

\section{Inference}

In the above graph, it shows that $30 \%(75-100)$ of workers showed no role limitations due to physical health, $33.3 \%$ (50-75) workers showed mild affection of role limitations, $25 \%$ workers (25-50) showed moderate affection of limitations, $11.6 \%(0-25)$ workers showed severe affection of role limitations due to physical health.

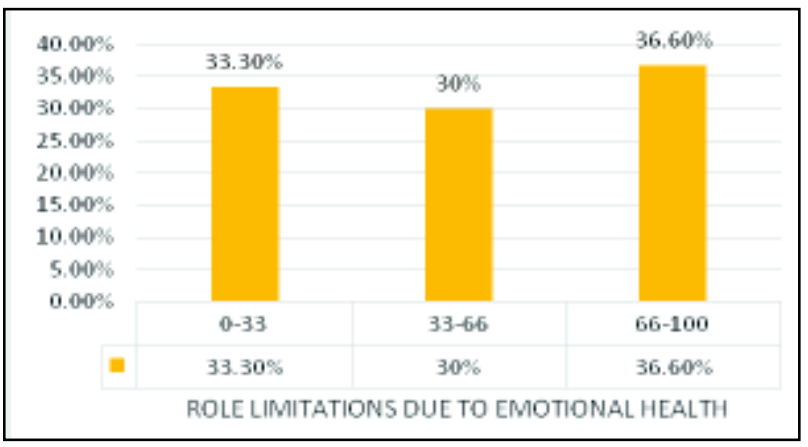

Graph 3: Role Limitations Due to Emotional Health

\section{Inference}

In the above graph, it shows that $36.6 \%$ (66-100) workers had mild affection of role limitations due to emotional health, 30\% (33-66) had moderate affection of role limitations while $33.3 \%$ showed severe affection of role limitations due to emotional health. 
PRABHU ET. AL.: QUALITY OF LIFE IN CONSTRUCTION SITE WORKERS

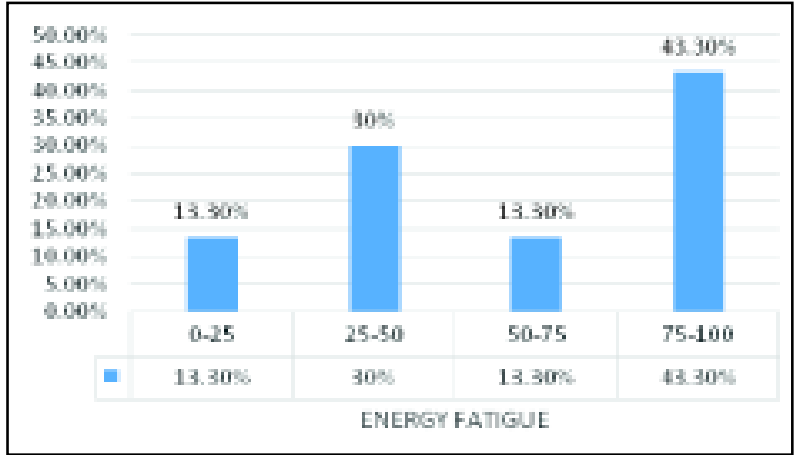

Graph 4: Energy Fatigue

\section{Inference}

In the above graph it showed that $43.30 \%$ workers (75-100) showed no energy fatigue. $13.30 \%$ workers (50$75)$ showed mild affection of energy fatigue, $30 \%(25-50)$ showed moderate affection of energy fatigue while $13.3 \%$ workers $(0-25)$ showed severe affection of energy fatigue.

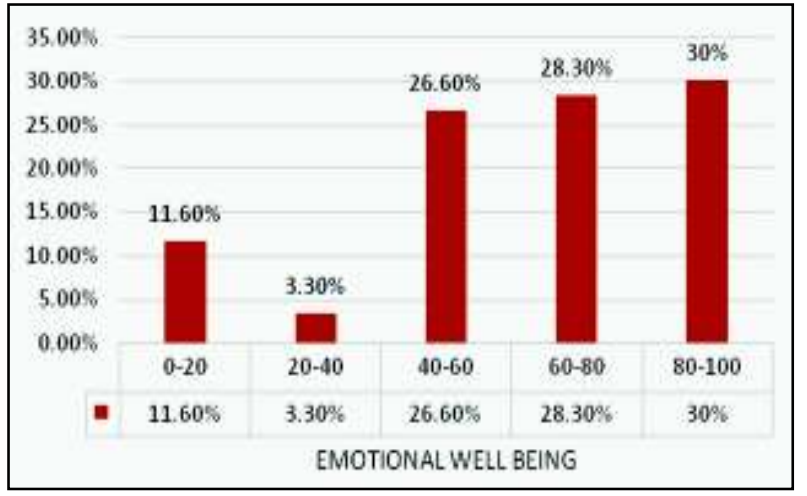

Graph 5: Emotional Well Being

\section{Inference}

In above graph it shows that $30 \%(80-100)$ had no affection in emotional well-being, $28.3 \%$ (60-80) had mild to no affection, $26.6 \%(40-60)$ had mild affection in emotional well-being, 3.3\% (20-40) had moderate affection while $11.6 \%(0-20)$ workers had severe affection in emotional well-being.

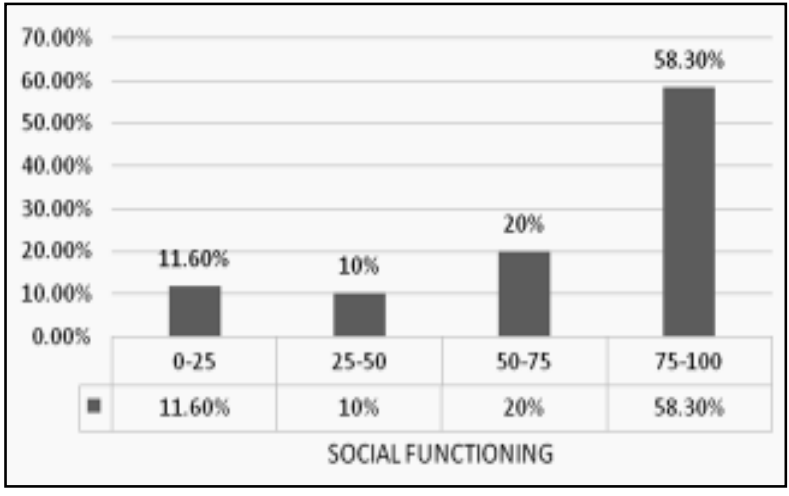

Graph 6: Social Functioning

\section{Inference}

In the above graph it shows that $58.3 \%$ (75-100) workers had no limitations in social functioning, $20 \%$ workers (50-75) had mild affection, 10\% (25-50) workers had moderate affection and $11.6 \%$ workers $(0-25)$ had severe affection in social functioning.

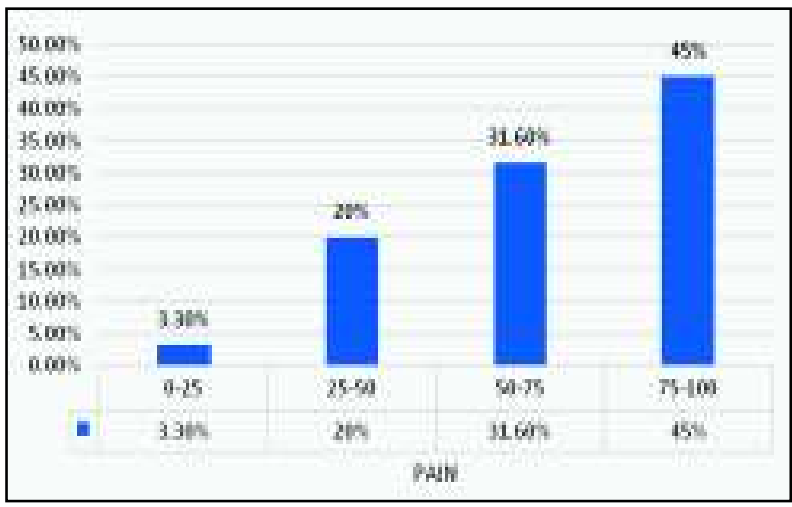

Graph 7: Pain

\section{Inference}

In the above graph it shows that $45 \%$ (75-100) workers had mild to nobody pain, $31.6 \%$ workers (50-75) had mild body pain, 20\% (25-50) workers had moderate body pain and $3.3 \%(0-25)$ had severe body pain. 


\section{PRABHU ET. AL.: QUALITY OF LIFE IN CONSTRUCTION SITE WORKERS}

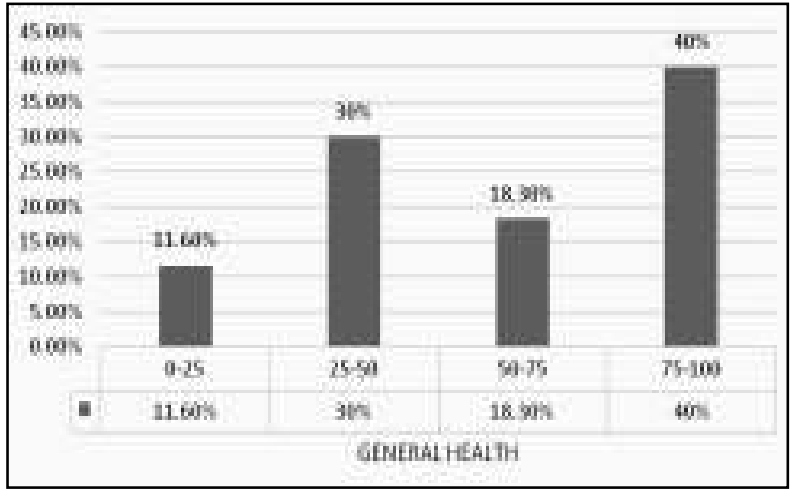

Graph 8: General Health

\section{Inference}

In the above graph, it shows that $40 \%$ (75-100) of workers health is excellent, $18.3 \%(50-75)$ workers health is good, $30 \%(25-50)$ workers health is fair while $11.6 \%(0-$ $25)$ workers health is poor.

\section{Discussion}

According to the graph-1 plotted, 25\% workers had no limitations in physical functioning, $23.8 \%$ workers had mild to no affection of physical functioning, 25\% workers had mild limitations, $21.6 \%$ workers had moderate limitations. Workers had mild to moderate limitations in vigorous activities, moderate activities like running, lifting heavy objects, climbing stairs, bending, kneeling, buying groceries, walking a mile, bathing or dressing themselves. 5 $\%$ had severe limitations in all these activities.

As per graph 2, 30\% of workers showed no role limitations due to physical health, $33.3 \%$ workers showed mild affection of role limitations, $25 \%$ workers showed moderate affection of limitations, where workers had cut down on their amount of time they spend on their work due to their physical health and had difficulty in performing their daily activities. $11.6 \%$ workers showed severe affection of role limitations due to their physical health.

According to graph 3, 36.6\% (66-100) workers had mild affection of role limitations due to emotional health, 30\% (33-66) had moderate affection of role limitations where they have cut down amount of time they spend on work due to their emotional problems while $33.3 \%$ showed severe affection of all role limitations due to emotional health.
According to graph 4, It showed that $43.30 \%$ workers (75-100) showed no energy fatigue. $13.30 \%$ workers (50-75) showed mild affection of energy fatigue,30\% (25-50) showed moderate affection of energy fatigue where they had felt loss of energy and tired while $13.3 \%$ (0-25) showed severe affection where they had severe loss of energy and felt tired.

According to graph 5, 30\% (80-100) had no affection in emotional well-being, $28.3 \%(60-80)$ had mild to no affection, $26.6 \%(40-60)$ had mild affection in emotional well-being, 3.3\% (20-40) had moderate affection in being nervous, down hearted and emotionally distressed while $11.6 \%(0-20)$ workers had severe affection in emotional well-being. Limitations in emotional domain could be due to working for longer hours in poor conditions with low wages and no safety precautions which prone to more physical injury and occupational hazards.

According to graph 6, 58.3\% (75-100) workers had no limitations in social functioning, $20 \%$ workers $(50$ 75 ) had mild affection, $10 \%$ (25-50) workers had moderate affection while being with their family or social activities due to their physical \& emotional pain and $11.6 \%$ workers $(0-25)$ had severe affection in social functioning can be due to emotional stress of work and physical pain which affects their social life with family.

According to graph 7, 45\% workers had mild to no body pain, $31.6 \%$ workers had mild body pain, $20 \%$ workers had moderate body pain and 3.3\% had severe body pain in the past 4 weeks where their pain interfered with their daily work. Prevalence of back (55\%), shoulder (32.5\%) is common among worker shaving pain, followed by neck \& knee $(30 \%)$ and the least pain was observed in calf $(10 \%)$ and ankle $(7.50 \%)$. Limitations in physical domain could be attributed to the fact that loss of functional activity could lead to disability. Most common pain observed was low back pain among workers (55\%). The main cause for LBP is due to the positions attained during work such as prolonged standing, bending, squatting and faulty lifting techniques. Most of the times spine is in the flexed position during the working hours due to this there is localized fatigue in the spinal musculature and stress on posterior longitudinal ligament. Constant posture also leads to spasm of spinal muscles and due to fatigue and spasm, pain is also present. Increase in the muscle activity also causes increase in the intra discal pressure. The increased pressure causes 


\section{PRABHU ET. AL.: QUALITY OF LIFE IN CONSTRUCTION SITE WORKERS}

redistribution of hydrostatic fluid and in turn causes stretching of annulus fibrosis, which affects posterior longitudinal ligament.

During a stoop lift, trunk flexion is achieved by thora columbar flexion and there is little to no knee flexion. During a squat lift, the spine remains as erect as possible and trunk flexion is achieved by hip \& knee flexion. Wilke and Nachemson found that inter vertebral disc pressures were higher when the load was held in stooped position than a squat position. The forces are increased when lifting. It has been documented that prolonged constrained standing compresses the inter vertebral disc, reduces disc hydration and reduces the inter vertebral distance between the adjacent vertebra and increasing the load on the facet joint. Inadequate rest pauses in between the working hours also increases load on the spine giving rise to low back pain.

Shoulder pain was next common pain observed in $32.5 \%$ workers. In workers lifting heavy weights and performing repeated movements of the arm leads stress and strain on shoulder joint. This job also has unique risk factors such as repetition, high force, awkward joint position, direct pressure, vibration and prolonged constrained posture. When lifting the weight the shoulder remains in state of flexion and abduction for most of the times. This leads to excessive tension on the shoulder stabilizers and shoulder girdle musculature. Inadequate work rest cycles, excessive pace or duration of work, unaccustomed work and lack of task variability and machine paced work can aggravate work related risk factors. Due to prolonged maintenance of this shoulder position there is a tendency for formation of micro- ruptures and adhesion that can occur which can lead to shoulder pain in these subjects. While performing overhead activities the deltoid and trapezius act continuously resulting into fatigue pain.

$30 \%$ workers had neck and knee pain. Neck pain can be while performing overhead activities the workers need to flex their neck and shrug their shoulder instead of maintaining it erect putting unnecessary strain on muscles around cervical region resulting in pain around the neck. Since construction workers lift heavy weights for $10-12 \mathrm{hrs}$ in day, there may be relative weakness of serratus anterior, middle and lower fibers of trapezius, rhomboids, posterior deltoid, upper thoracic extensors, deep flexors and shoulder rotators.
Knee pain can be due to long period of standing and bending, stress is applied to the knee joint which is the weight bearing joint and is prone to degenerative conditions. Due to faulty postures and constant work in one position tightening of hamstrings and calf muscles occur. Due to faulty alignment of muscle fibers they go into shortening and limit ROM and are associated with pain. Weakness and fatigue also contributes to pain. Knee pain was found to be more common in older age group as some amount of degenerative changes may also have started to appear with age. $10 \%$ workers had calf pain and $7.5 \%$ ankle pain due to prolonged standing, static posture leads to sustained contraction of the calf muscles.

According to the graph 8 , It shows that $40 \%$ (75$100)$ of workers health is excellent, $18.3 \%$ (50-75) workers health is good, $30 \%(25-50)$ workers health is fair while $11.6 \%(0-25)$ workers health is poor due to adaptation to poor quality of life.

$33.3 \%$ workers had severe role limitations due to emotional health where they had to cut down amount of time they spend on work due to their emotional problems. The domain which is maximally affected is role limitations due to emotional health, followed by energy fatigue, emotional well-being, social functioning, general health, role limitations due to physical health, physical functioning and least affected domain was affected pain.

According to the domains:

1. In role limitations due to emotional health, $36.6 \%$ workers had mild limitations while $33.3 \%$ workers had severe role limitations due to emotional health.

2. In Energy fatigue, $43.3 \%$ workers had no loss of energy while $13.3 \%$ workers had severe loss of energy.

3. In emotional well-being, $30 \%$ workers had no limitations in emotional well-being while $11.6 \%$ workers had severe affection in emotional wellbeing.

4. In social functioning, $58.3 \%$ workers had no limitations while $11.6 \%$ workers had severe limitations in social functioning.

5. In general health, $40 \%$ workers general health is excellent while $11.6 \%$ workers general health is poor.

6. In role limitations due to physical health, 30\% workers had no limitations while $11.6 \%$ workers had severe role limitations due to physical health. 
7. In physical functioning, $25 \%$ workers had no limitations while $5 \%$ workers had severe limitations of physical functioning.

8. In pain, $45 \%$ workers had mild to no pain affection while $3.3 \%$ had severe body pain.

\section{AKNOWLEDGEMENTS}

I take this opportunity to express my sincere gratitude to those people without whose support and concern this project would not have been a success.I am also extremely thankful to Dr. Unnati Pandit, Director and professor, who has given me the permission to initiate the project in this Institution and has give nher valuable advice and help throughout. I am extremely thankful to my guides Dr. Sukhada Prabhuand Dr. Manasi Desai for their valuable guidance, for giving their valuable time and help throughout. And last but not the least, the colleagues of our branch and my study subjects who deserve a word of thanks for their co-operation.

\section{REFERENCES}

Ahmad A. and Shahnawaz A., 2015. Prevalence of Musculoskeletal Pain In Construction Workers In Saudi Arabia. The Scientific World Journal.

Connell J., Brazier J. and Paisley S., 2012. Quality of life of people with mental health problems: synthesis of qualitative research. Health Qual life outcomes, 10:138.

Dhar R.L., 2014. Understanding Working Class Lives: An Examination Of Quality Of Life Of Low Income Construction Workers, 49(1):87-105.

Eaves S., 2016. Building Healthy Construction Workers, Their views on Health, Well Being and Better Work Place Design, Applied Ergonomics, Volume 54.
Fawkes C., 2013. Sf-36 introduction, NCOR.

Jacobsen H., 2014. Construction Workers Struggle With High Prevalence of Mental Distress and It is Associated With Their Pain and Injuries, J. Occup environ. Med. journal.

Jacobsen H.B., 2013. Construction Workers Struggle With a High prevalence of mental distress, and this is associated with their pain and injuries.Et al., J. Occup Environ. Med.

Kinetics and Kinematics of Lumbar Spine, Lumbar Pelvic Rhythm, Joint Structure and Function, Pamela k levangie and Cynthia norkins, $5^{\text {th }}$ edition.

Laskar A. and Murthy C.V.R., 2003. Challenges Before Construction Industry In India. Department of Civil Engineering.

Matthew G., Ramesh N. and Desai P., 2016. Quality Of Life And Probable Psychological Distress Among Male Workers At a Construction Site, Kolar District, Karnataka Public Indian J. Occup Environ. Med. Journal.

Nag A., Vyas H. and Nag P., 2016. Occupational Health Scenario of Indian and Informal Sector. Ind. Health, 54(4): 377-385.

Oliver Lc et al.,, 2006. Airway disease in highway and tunnel construction workers exposed to silica. Am. J. Ind. Med.

Qu B. et al.,, 2009. Reliability And Validity Testing Of the SF 36 Questionnaire for the Evaluation Of the Quality of life of Chinese urban Construction Workers. J. Int. Med. Res. 